\title{
Poly- and Monoamine Metabolism in Streptomyces coelicolor: The New Role of Glutamine Synthetase-Like Enzymes in the Survival under Environmental Stress
}

\author{
Sergii Krysenko ${ }^{a} \quad$ Arne Matthews $^{\mathrm{a}}$ Tobias Busche ${ }^{\mathrm{b}}$ Agnieszka Bera ${ }^{\mathrm{a}}$ \\ Wolfgang Wohlleben ${ }^{a}$ \\ aDepartment of Microbiology and Biotechnology, Interfaculty Institute of Microbiology and Infection Medicine \\ Tübingen (IMIT), Cluster of Excellence 'Controlling Microbes to Fight Infections', University of Tübingen, Tübingen, \\ Germany; ${ }^{b}$ Center for Biotechnology (CeBiTec), Bielefeld University, Bielefeld, Germany
}

\author{
Keywords \\ Nitrogen assimilation · GS-like enzyme · GlnA • GInA2 • \\ GlnA3 · GInA4 · Polyamine metabolism · Ethanolamine \\ metabolism $\cdot$ S. coelicolor
}

\begin{abstract}
Soil bacteria from the genus Streptomyces, phylum Actinobacteria, feature a complex metabolism and diverse adaptations to environmental stress. These characteristics are consequences of variable nutrition availability in the soil and allow survival under changing nitrogen conditions. Streptomyces coelicolor is a model organism for Actinobacteria and is able to use nitrogen from a variety of sources including unusual compounds originating from the decomposition of dead plant and animal material, such as polyamines or monoamines (like ethanolamine). Assimilation of nitrogen from these sources in S. coelicolor remains largely unstudied. Using microbiological, biochemical and in silico approaches, it was recently possible to postulate polyamine and monoamine (ethanolamine) utilization pathways in $S$. coelicolor. Glutamine synthetase-like enzymes (GS-like) play a central role in these pathways. Extensive studies have revealed that these enzymes are able to detoxify polyamines
\end{abstract}

or monoamines and allow the survival of $S$. coelicolor in soil containing an excess of these compounds. On the other hand, at low concentrations, polyamines and monoamines can be utilized as nitrogen and carbon sources. It has been demonstrated that the first step in poly-/monoamine assimilation is catalyzed by $\mathrm{G} \ln \mathrm{A} 3$ (a $\gamma$-glutamylpolyamine synthetase) and GInA4 (a y-glutamylethanolamide synthetase), respectively. First insights into the regulation of polyamine and ethanolamine metabolism have revealed that the expression of the $g \ln A 3$ and the glnA4 gene are controlled on the transcriptional level.

(C) 2021 The Author(s) Published by S. Karger AG, Basel

\section{Introduction}

Streptomyces coelicolor is a filamentous soil-dwelling, non-motile, Gram-positive, obligatory aerobe bacterium with high GC-content (63-78\%) and belongs to the genus Streptomyces from the phylum Actinobacteria [Bentley et

Arne Matthews' present address: Faculty of Biology, University of Freiburg, Freiburg, Germany karger@karger.com www.karger.com/mip

Karger $\stackrel{\text { ' }}{5}$

BOPEN ACCESS
(C) 2021 The Author(s)

Published by S. Karger AG, Basel

This is an Open Access article licensed under the Creative Common Attribution-NonCommercial-4.0 International License (CC BY-NC) (http://www.karger.com/Services/OpenAccessLicense), applicable to the online version of the article only. Usage and distribution for commercial purposes requires written permission.
Correspondence to:

Wolfgang Wohlleben, wolfgang.wohlleben@ biotech.uni-tuebingen.de 
al., 2002]. Streptomycetes including S. coelicolor feature a complex life cycle and metabolism, the ability to synthesize useful natural products, high metabolic potential as well as impressive adaptability to environmental stress [Hopwood et al., 1995].

In its natural habitat, the soil, $S$. coelicolor can live under numerous nutrient conditions. It is able to metabolize varying $\mathrm{N} / \mathrm{C}$-sources, including amino acids, peptides, amino sugars, ammonium $\left(\mathrm{NH}_{4}{ }^{+}\right)$, nitrate $\left(\mathrm{NO}_{3}{ }^{-}\right)$, nitrite $\left(\mathrm{NO}_{2}{ }^{-}\right)$, and urea [Hodgson, 2000]. Members of the family Streptomycetaceae fulfil an important ecological role in the soil due to their capability to metabolize the remains of other organisms. For instance, they are able to detoxify and utilize different $\mathrm{C}$ - and $\mathrm{N}$-sources (chitin, xylose and cellulose) as well as to release chitinases, xylanases, and cellulases for extracellular utilization of organic material [Hodgson, 2000].

In terms of genetics, physiology, and applications, $S$. coelicolor A3(2) is the best studied and described actinobacterium and, therefore, serves as a model organism for Streptomyces and Actinobacteria [Hopwood, 1999]. The genome of the widely used S. coelicolor M145 strain was fully sequenced and is remarkably large. This strain lacks two original S. coelicolor A3(2) plasmids SCP1 and SCP2 and contains a linear chromosome with $8,667,507$ base pairs corresponding to 7,825 predicted genes [Bentley et al., 2002]. The genome of $S$. coelicolor includes multiple gene clusters for natural product synthesis, for example undecylprodigiosin, actinorhodin, calcium-dependent antibiotic, methylenomycin, perimycin [Hopwood, 2006]. Primary and secondary metabolism of S. coelicolor as well as morphological differentiation are controlled by interlinked regulatory networks that respond to the variety of intra- and extracellular stimuli, including signals concerning the nitrogen availability and supply [Karandikar et al., 1997]. These features allow S. coelicolor to be very competitive in soil and to survive under environmental stress.

\section{Nitrogen Metabolism in S. coelicolor}

\section{Assimilation of Nitrogen in S. coelicolor}

Nitrogen from the extracellular environment can be assimilated via various utilization pathways. Nitrogen control has been studied in a number of bacteria, including the actinobacterial species Corynebacterium glutamicum, Mycobacterium tuberculosis, and S. coelicolor [Merrick et al., 1995].
For S. coelicolor, common nitrogen sources in soil environment are ammonium, glutamine, glutamate, and nitrate $\left(\mathrm{NO}^{-}\right)$. The amino acids histidine and arginine were also shown to be preferable $\mathrm{N}$-sources for S. coelicolor [Hodgson, 2000; Hopwood, 2006]. These compounds can diffuse through the membrane (ammonium) or are taken up directly by transporters (ammonium, nitrate/nitrite, urea, amino acids) [Thomas et al., 2000]. Ammonium is a preferred inorganic nitrogen source and can be directly incorporated into glutamine or glutamate, which are important building blocks for biomolecules. Other nitrogen sources have to be transformed first, for example cleaved like urea or deaminated/transaminated-like amino acids and are then converted to ammonium [Fischer et al., 2010] (shown in Fig. 1).

Under low N-concentrations, the intracellular ammonium is introduced into the metabolism by glutamine synthetase (GS), which catalyzes the synthesis of glutamine using ammonium and glutamate as substrates in an ATP-dependent manner. Glutamate can be produced from glutamine and 2-oxyglutarate by glutamine-2-oxoglutarate-aminotransferase (GOGAT) [Magasanik, 1982]. Under high N-concentrations, glutamate dehydrogenase $(\mathrm{GDH})$ catalyzes the production of glutamate from ammonium and 2-oxyglutarate using NADPH (shown in Fig. 1). These basic reactions are also conserved in $S$. coelicolor, which possesses as a distinctive feature two GSs (GSI-GlnA and GSII-GlnII) that are able to synthesize glutamine (see section GS-Like Enzymes in S. coelicolor).

\section{Regulation of Nitrogen Assimilation in S. coelicolor}

The cellular response to nitrogen limitation on the transcriptional level is controlled in S. coelicolor by the global nitrogen response regulator GlnR from the OmpR family of response regulators [Wray \& Fisher, 1993]. It is an atypical orphan response regulator, which influences the expression of genes that are directly involved in nitrogen assimilation, e.g. the operon $a m t B-g \ln K-g \ln D$, encoding the ammonium transporter AmtB, the PII signal protein $\mathrm{GlnK}$, and the adenylyl transferase GlnD [Reuther \& Wohlleben, 2007; Tiffert et al., 2008]. These genes are conserved across the order Actinomycetales [Fink et al., 2002; Tiffert et al., 2008]. Other GlnR target genes include nirB and nas $A$ for nitrate/nitrite reduction, ureA for cleavage of urea, $g \ln A$ and $g \ln I I$ encoding glutamine synthetases, $g d h A$ encoding a glutamate dehydrogenase, $n n a R$ encoding the HemD-like transcriptional regulator and seven additional genes encoding proteins of unknown function [Reuther \& Wohlleben, 2007; Tiffert et 
Fig. 1. Schematic illustration of nitrogen metabolism in S. coelicolor. GS, glutamine synthetase; GDH, glutamate dehydrogenase; GOGAT, glutamine-2-oxoglutarateaminotransferase.

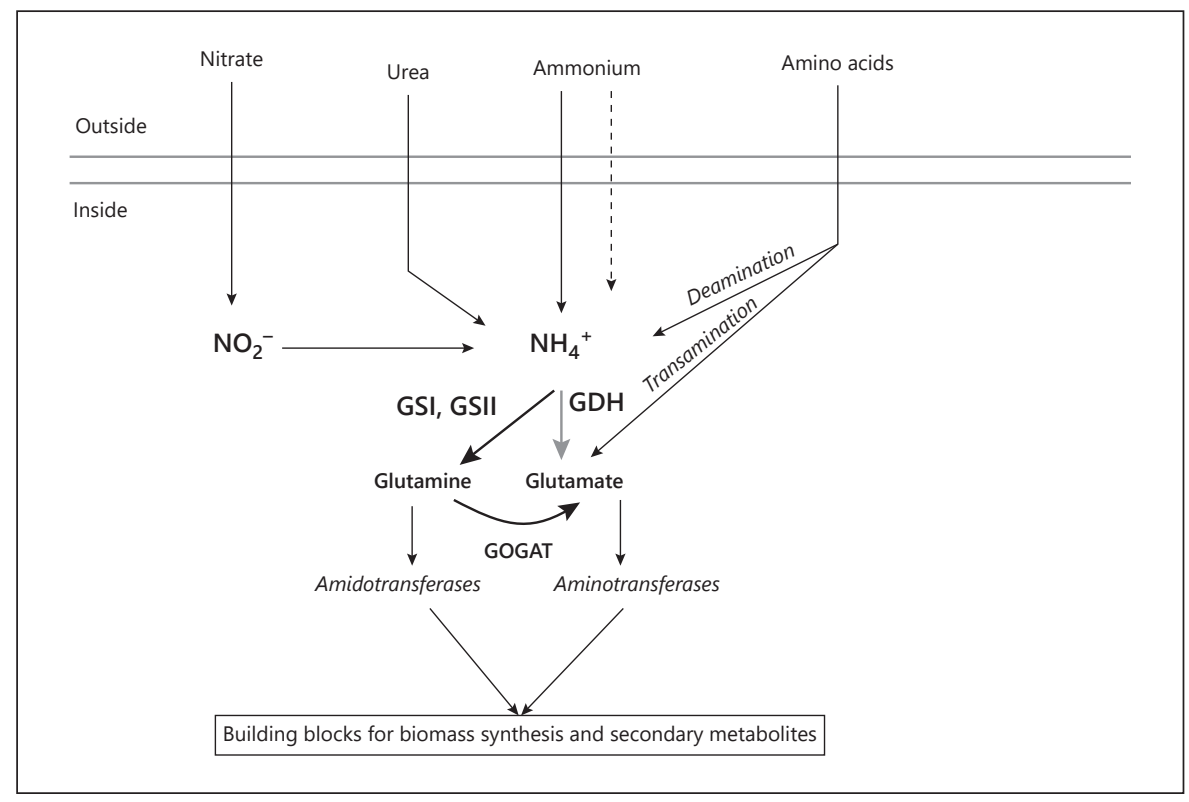

al., 2008; Wang \& Zhao, 2009;Amin et al., 2012]. GlnR can be modified by phosphorylation and acetylation under $N$-variable conditions. Such post-translational modifications can change GlnR DNA-binding activity [Amin et al., 2016].

Transcriptional control of the nitrate assimilatory genes (nnaR, narK, nas A, nirBD) in S. coelicolor involves, in addition to GlnR, a second regulator NnaR. Under nitrogen limitation conditions, GlnR activates the expression of nitrate assimilatory genes that are enhanced by the synergistic binding of $\mathrm{NnaR}$ and $\mathrm{GlnR}$ in the presence of nitrate [Amin et al., 2012]. One more regulator GlnRII exhibits $31 \%$ amino acid sequence identity to $G \ln R$ and shows the same binding specificity as GlnR. GlnRII can also bind to the upstream regions of $g \ln A, g \ln I I, a m t B$ $g \ln K-g \ln D$, and sco1863. However, the deletion of $g \ln R I I$ has been shown not to result in glutamine auxotrophy, meaning that GlnRII is not a functional homologue of GlnR. Since an in vitro interaction of GlnRII with the $g \ln I I$ promoter has been demonstrated, this regulator probably has a particular role in the regulation of $g \ln I I$ [Fink et al., 2002]. GlnRII was found in a large number of screened Streptomyces strains, but has not been found in Mycobacterium or Corynebacterium [Reuther \& Wohlleben, 2007]. The GlnR/GlnRII system may have been evolved to allow an efficient differential transcription control of both GS genes $g \ln A$ and $g \ln I I$. In $S$. coelicolor, GSI is predominantly responsible for primary metabolism, while GSII may be particularly involved in secondary metabolism [Hillemann et al., 1993; Reuther \& Wohlleben, 2007].

Poly- and Monoamine Metabolism in Streptomyces coelicolor
Depending on the conditions, the complex regulation of nitrogen metabolism in S. coelicolor involves additional control by other regulators such as PhoP [Rodríguez-García et al., 2009; Sola-Landa et al., 2013], Crp [Gao et al., 2012], ArgR [Perez-Redondo et al., 2012], AfsR [SantosBeneit et al., 2012], and AfsQ1 [Wang et al., 2013]. Under phosphate limitation, PhoP negatively controls the transcription of $g \ln R, g \ln A, g \ln I I$ as well as $a m t B-g \ln K-g \ln D$ [Rodríguez-García et al., 2009]. Crp positively controls $g \ln A, g \ln I I$, and $a m t B-g \ln K-g \ln D$ (but not $g \ln R$ ). Also, this regulator controls the interface of primary and secondary metabolism [Gao et al., 2012]. AfsR regulates expression of $g \ln R$ in response to an unknown nutrient stress stimulus [Santos-Beneit et al., 2012]. AfsQ1 is involved in the regulation of the carbon, nitrogen, and phosphate metabolism in the presence of glutamate [Wang et al., 2013]. Additionally, AfsR and AfsQ1 are implicated in the control of secondary metabolite production.

Regulation of nitrogen metabolism in S. coelicolor involves additional post-translational control. Depending on the ammonium level, ammonium uptake is post-translationally controlled by the interaction of the nitrogen sensor protein PII (GlnK) with the ammonium transporter (AmtB). Under high ammonium concentrations, GlnK is inactivated by the GlnD (adenylyltransferase)-mediated adenylylation or specific proteolysis [Fink et al., 2002]. Furthermore, the activities of glutamine synthetases are controlled at the post-translational level. In response to changing nitrogen conditions, the activity of GlnA has been shown to be regulated through reversible adenyl- 
Fig. 2. Schematic illustration of the posttranslational regulation of nitrogen metabolism in S. coelicolor. GSI, glutamine synthetase I; AMP, adenosine monophosphate; UMP, uridine monophosphate; question mark, unknown regulatory protein [modified after Fink et al., 2002; Reuther \& Wohlleben, 2007].

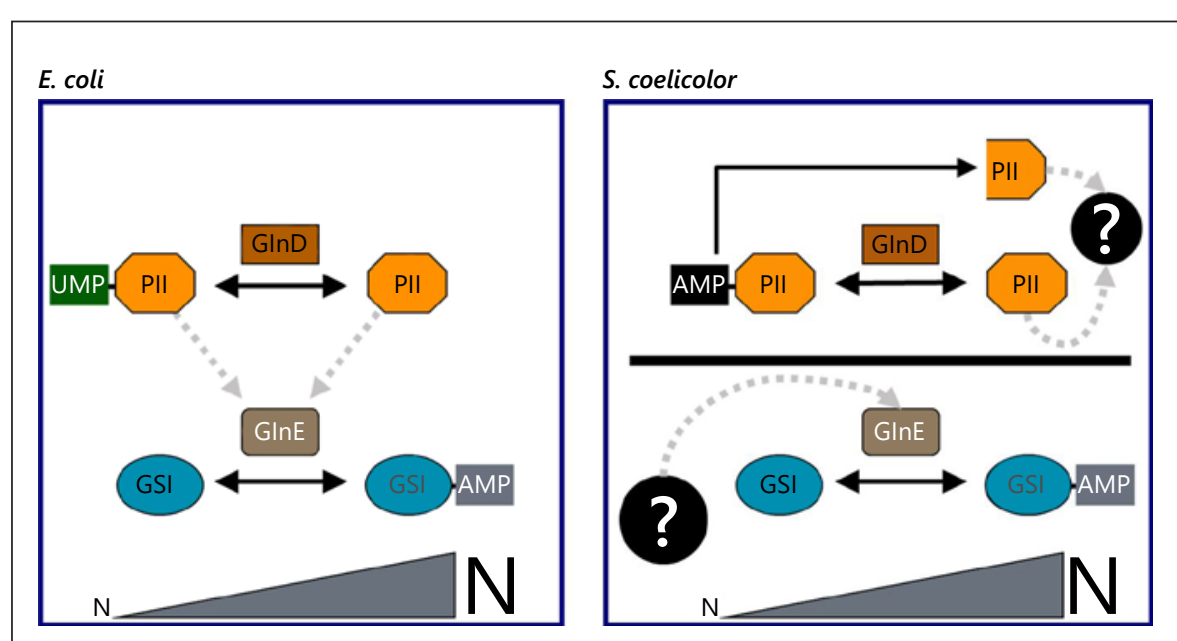

Fig. 3. 3D structures of GSI, GSII, and GSIII proteins. a The structure of GlnA from S. coelicolor (Swiss-Model based on 1FPY template). b The crystal structure of GlnII from S. coelicolor (PDB entry 4BAX by K. Zeth). c The crystal structure of GSIII from B. fragilis (PDB entry 3O6X by J.M. van Rooyen). Color scheme: chain (each protein chain has its own colour). Dashed arrows point out the catalytic centre of the enzyme, representing one of twelve catalytic centres between each monomer.

ylation and deadenylylation by an adenylyltransferase GlnE [Fink et al., 1999], but there have been no reports of post-translational modification of the GOGAT enzyme [Harper et al., 2008]. A GlnE-mediated regulation of GSI was first shown in enterobacteria like Escherichia coli, where $\mathrm{Gln} E$ is regulated by the bicyclic regulatory cascade involving $\mathrm{GlnD}$ and $\mathrm{GlnB}$ (nitrogen regulatory protein P-II 1) [Magasanik 1982; Ninfa et al., 2001]. However, in S. coelicolor, the PII protein GlnK as well as the adenylyltransferase $\mathrm{Gln} D$ are not essential for the GlnE-dependent GSI regulation, since it also occurs in the $g \ln K$ and $g \ln D$ deletion mutants [Hesketh et al., 2002] (shown in Fig. 2).

\section{Glutamine Synthetases of S. coelicolor}

Glutamine synthetases (GS; EC 6.1.1.3) are ubiquitous, homo-oligomeric enzymes, which play a central role in nitrogen assimilation in all forms of life [Pesole et al., 1995]. Glutamine synthetases were hypothesized to be among the most ancient existing enzymes [Kumada et al., 1993] and are not restricted to a specific domain of life. Three distinct types of GS have been described. The GS type-1 (GSI) has been found in all prokaryotes, and also in mammals and plants [Merrick \& Edwards, 1995]. GS type-2 (GSII) has been found in eukaryotes and like GSI also in some soil bacteria, for example Rhizobium sp., 


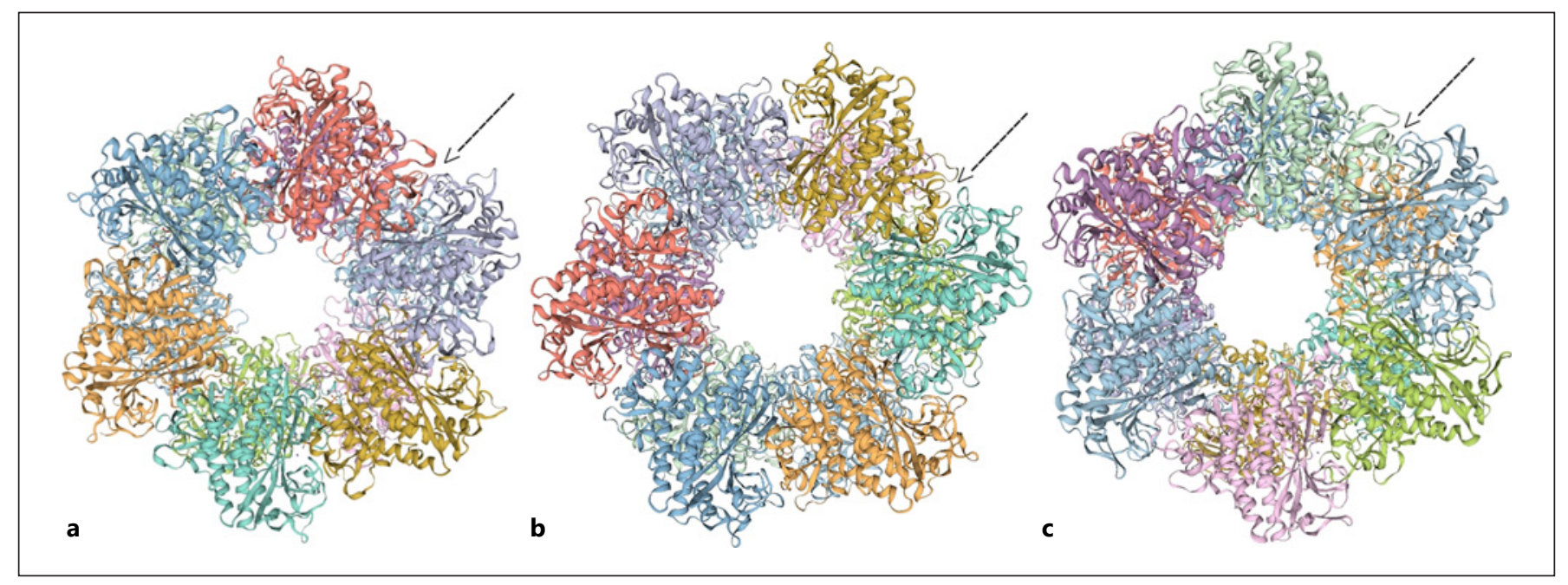

Fig. 4. 3D structures of GS-like proteins from S. coelicolor generated via homology modelling using Swiss-Model tool and based on the 1FPY template. $\mathbf{a}$ the structure of GlnA2; b: the structure of GlnA3; c: the structure of GlnA4. Color scheme: chain (each protein chain has its own colour). Dashed arrows point out the catalytic centre of the enzyme, representing one of twelve catalytic centres between each monomer.

Frankia sp., Streptomyces sp. [Behrmann et al., 1990; Kumada et al., 1993; Merrick \& Edwards, 1995]. GS type-3 (GSIII) has been found in the anaerobe Gram-negative bacterium Bacteriodes fragilis, in cyanobacteria and some protozoans [Pesole et al., 1995].

Structurally, GSs are composed of two closed ring structures (with active sites formed between protomers). GSI are dodecamers, GSII are decamers, GSIII are dodecamers, while GSIII are much larger compared to GSI and GSII [Kumada et al., 1993; Eisenberg et al., 2000]. Each active site creates a binding pocket with distinct substrate binding sites for a nucleotide (ATP), amino acid (glutamate), ammonium ion, as well as two sites for divalent cations $\left(\mathrm{Mn}^{+2}\right.$ or $\left.\mathrm{Mg}^{+2}\right)$ [Eisenberg et al., 2000] (shown in Fig. 3).

In S. coelicolor, GlnA consists of 12 subunits and reveals high sequence similarity to other prokaryotic GSI enzymes [Wray \& Fisher, 1988]. $g \ln A$ is transcribed during all growth phases [Fink et al., 2002]. GlnII is a homodecamer and possesses high sequence similarity to eukaryotic GSII enzymes [Hillemann et al., 1993]. The glnII gene was detected in all Streptomyces species tested, but not in other actinobacteria, like Mycobacterium and Corynebacterium [Behrmann et al., 1990]. In contrast to $g \ln A$ transcription, the expression level of the $g \ln I I$ transcript increases with the onset of mycelial differentiation [Fink et al., 2002], suggesting a role of GlnII in late growth phases.

Poly- and Monoamine Metabolism in Streptomyces coelicolor
Structural Analysis of the Glutamine Synthetase GlnII from S. coelicolor

The crystal structures of different types of glutamine synthases have previously been elucidated and characterized in such bacteria as Salmonella typhimurium (PDB entry 1LGR (GSI); PDB entry 1FPY (GSI)), Helicobacter pylori (PDB entry 5ZLI (GSI)), Bacillus subtilis (PDB entry 3QAJ (GSI)), Bacteroides fragilis (PDB entry 3O6X (GSIII)) and M. tuberculosis (PDB entry 1HTO (GSI)). $\mathrm{X}$-ray diffraction was used to determine the crystal structure of GSII (GlnII) in S. coelicolor with a resolution 2.55 $\AA$ (released in PDB as 4BAX). The GSII of S. coelicolor comprises 10 identical subunits organized in 2 rings. The biologically relevant decamer contains 10 active sites between each monomer. Similar to known GlnA structures, GlnII features a tunnel created by each active site, which contains three substrate binding sites for the ATP, glutamate and ammonium. In the middle, divalent cations of $\mathrm{Mn}^{+2}$ or $\mathrm{Mg}^{+2}$ can bind. Each ring of GlnII consists of 5 subunits that are held together through hydrogen bonding and hydrophobic interactions (shown in Fig. 3, b).

\section{GS-Like Enzymes in S. coelicolor}

Interestingly, an in silico analysis of $g \ln A$-genes across actinobacterial genomes demonstrated the existence of a hypothesized common ancestor of $g \ln A$ and $g \ln I I$. Apparently, the common ancestor has evolved to glutamine synthetase-encoding genes and subsequently to special- 
ized $g \ln A$-like genes $(g \ln A 2, g \ln A 3, g \ln A 4)$ [Kumada et al., 1993; Hayward et al., 2009].

In the model actinobacterium, $S$. coelicolor, three $g l$ $n A$-like genes were identified in addition to $g \ln A$ and $g l$ $n I I: g \ln A 2$ (sco2241), $g \ln A 3$ (sco6962), and $g \ln A 4$ (sco1613) [Rexer et al., 2006; Reuther \& Wohlleben, 2007]. Only GlnA and GlnII were shown to be functional glutamine synthetases [Krysenko et al., 2017]. Structural analysis of GS-like enzymes through homology modelling revealed that they are composed of 12 subunits in two rings with six subunits each, with an active site between each of two neighbouring subunits (shown in Fig. 3). Studies on GlnA2 (shown in Fig. 4, a) could show that it is involved in nitrogen metabolism and may be structurally more similar to GlnA (shown in Fig. 3, a) than GlnA3 and GlnA4, according to homology modelling (shown in Fig. 4, b and c). It has been shown that in S. coelicolor GlnA2 may play role in the post-translational regulation of the global nitrogen metabolism regulator GlnR and in the signal mediation of nitrogen excess to the adenyltransferase GlnE. Glutamine, synthesized by GlnA, seems to induce the expression of $g \ln A 2$, leading to elevated levels of $\mathrm{G} \ln \mathrm{A} 2$ in the cell. GlnA2 binds subsequently to GlnR and GlnE and influences their activity [Nentwich, 2012]. Nevertheless, GlnA2 has been interpreted to be an important signal mediator and regulator in nitrogen metabolism under high $\mathrm{N}$-concentrations; the role of $\mathrm{Gln} \mathrm{A} 2$ in nitrogen metabolism has remained poorly understood.

GlnA2/3/4 of $S$. coelicolor show moderate levels of amino acid sequence identity compared to $\mathrm{G} \ln \mathrm{A}$ (GlnA2: 32\% identity, 48\% similarity; GlnA3: 31\% identity, 50\% similarity; GlnA4: $27 \%$ identity, $41 \%$ similarity). Since GS-like proteins are not able to synthesize glutamine from ammonium and glutamate, it was hypothesized that they may not accept ammonium as substrate, but other nitrogen containing compounds, which are typically present in soil. A phenotypic analysis of $g \ln A 2, g \ln A 3$ and $g \ln A 4$ knockout mutants in the presence of different sole nitrogen sources finally revealed that GS-like enzymes can accept poly- and monoamines as substrates [Krysenko et al., 2017; 2019].

\section{Polyamine Metabolism in Bacteria}

\section{Distribution and Role of Polyamines in Bacteria}

Chemically polyamines are aliphatic polycations with a polycarbon chain and multiple amino groups. Commonly known and widely distributed natural polyamines present in all organisms are putrescine (1,4-diaminobu-

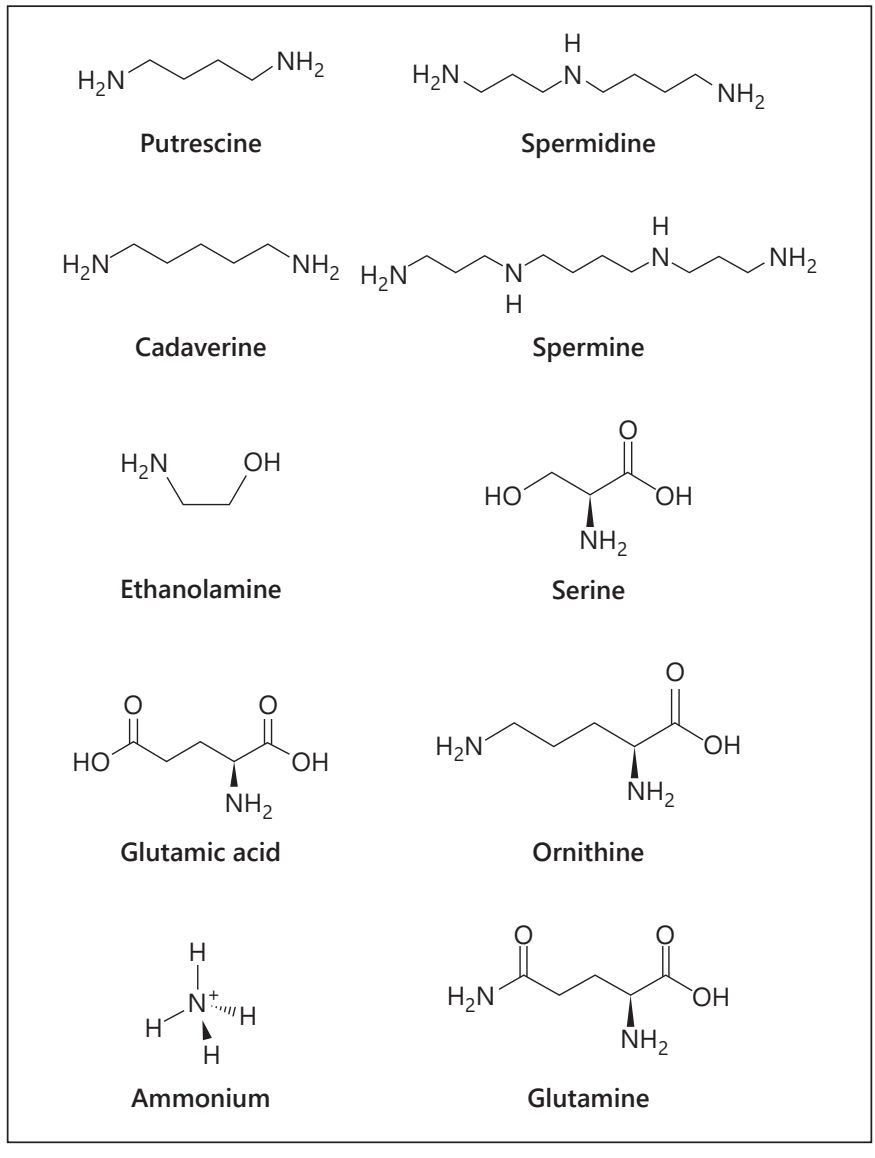

Fig. 5. Chemical formulae of common polyamines, ethanolamine, and selected amino acids.

tane), cadaverine (pentane-1,5-diamine), spermidine ( $\mathrm{N}$ (3 aminopropyl)-1, 4-butadiamine), and spermine ( $\mathrm{N}$, $\mathrm{N}^{\prime}$-bis (3-aminopropyl-1, 4-butanediamine)) [MillerFleming et al., 2015] (shown in Fig. 5). There are other, rarely occurring polyamines in nature, for example thermine, thermospermine, caldopentamine, and others described in the extreme thermophile Thermus thermophiles [Oshima et al., 1988].

Polyamines can be found in diverse bacterial habitats. For instance, in soil, the concentration of putrescine ranges between 0.28 and $0.56 \mathrm{nmol} / \mathrm{g}$, spermidine between 0.23 and $0.62 \mathrm{nmol} / \mathrm{g}$, and spermine between 0.16 and $0.43 \mathrm{nmol} / \mathrm{g}$. Increased polyamine concentrations lead to bacterial cell death [Young \& Chen, 1997].

Intracellular polyamine concentrations vary between species. For example, E. coli contains high concentrations of putrescine $(10-30 \mathrm{mM})$, while in most bacteria the intracellular content of putrescine ranges between 0.1 and $0.2 \mathrm{~mm}$ [Cohen, 1998]. In $\gamma$-proteobacteria, polyamine 


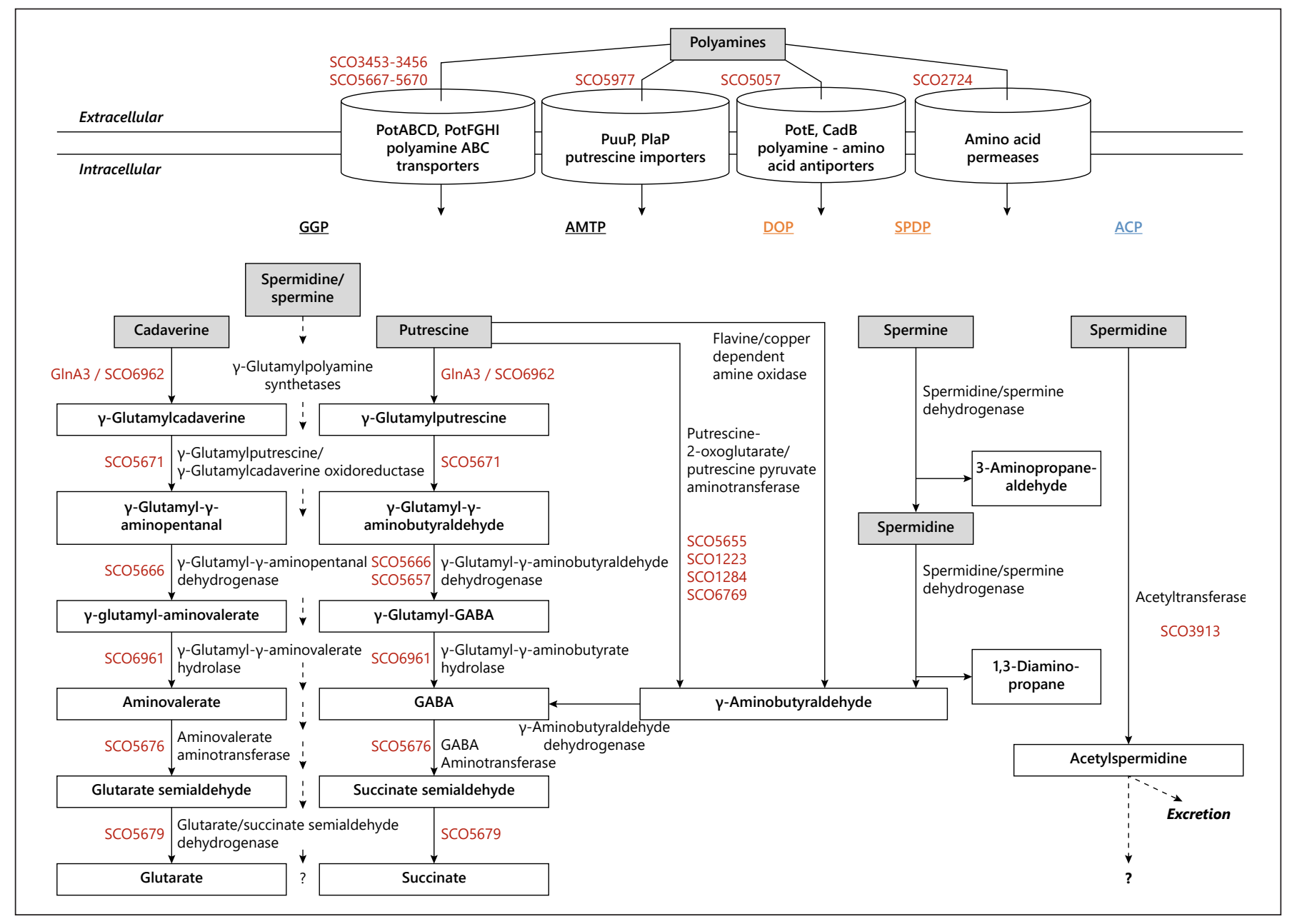

Fig. 6. Combined scheme of polyamine utilization pathways within prokaryotes [modified after Krysenko et al., 2017]. GGP, gamma-glutamylation pathway; AMTP, aminotransferase pathway; DOP, direct oxidation pathway; SPDP, spermine/spermidine dehydrogenase pathway; ACP, acetylation pathway. Dashed arrows represent predicted and straight arrows confirmed metabolic pathways. Pathways in black: E. coli and P. aeruginosa, red: $S$. coelicolor (bold: function confirmed - GlnA3, not bold: prediction), orange: P. aeruginosa, blue: B. subtilis.

depletion has been shown to reduce the growth rate, for example in E. coli [Chattopadhyay et al., 2009]. Spermidin was shown to be essential for growth of Gram-negative bacteria, like the $\gamma$-proteobacterium Pseudomonas aeruginosa PAO1 [Nakada \& Itoh, 2003]. Interestingly, in the Gram-positive bacterium Bacillus subtilis, polyamines were reported not to be required for normal growth [Burrell et al., 2010]. In contrast to putrescine and spermidine, the role of cadaverine and spermine in bacterial cells is still not well established.

Despite the fact that polyamines, especially spermidine, are known to be essential in eukaryotic and archaeal life, their role in bacteria still remains less clear. Intracellular polyamine levels have been shown to be tightly reg- ulated with cell metabolism. Polyamines were shown to be accumulated intracellularly during exposure to some stress conditions. In bacteria, the functions of polyamines include the regulation of transcription and translation, the cell growth stimulation and biofilm formation, acid resistance, the response to oxidative stress, biosynthesis of siderophores, SOS system activation, and antibiotic resistance [Miller-Fleming et al., 2005; Kusano \& Suzuki, 2015].

\section{Polyamine Biosynthesis and Uptake in Bacteria}

Polyamines can be synthesized predominantly from the amino acids ornithine and methionine, but also from arginine and lysine. Putrescine is usually formed by orni- 
thine decarboxylation through an ornithine decarboxylase, but it can also be synthetized from arginine via arginine decarboxylase and agmatinase [Miller-Fleming et al., 2015]. Spermidine and spermine are formed from putrescine via addition of aminopropyl groups, which are provided by decarboxylated S-adenosylmethionine (dcAdoMet, dSAM). dSAM is produced from S-adenosylmethionine by an S-adenosylmethionine decarboxylase (AdoMetDC, SpeD). The addition of aminopropyl groups is catalyzed by spermidine and spermine synthetases (SpdS/SpeE and SpmS) [Miller-Fleming et al., 2015; Kusano \& Suzuki, 2015]. Cadaverine is a product of lysine decarboxylation by lysine decarboxylases (LDCs) that have been studied in E. coli, Vibrio sp., and Lactobacillus sp. [Tomar et al., 2013].

In S. coelicolor, the biosynthesis and intracellular content of polyamines have barely been investigated. It was reported that putrescine and spermidine biosynthesis may occur in the late-stationary phase in minimal liquid medium, while cadaverine may be synthesized under iron limitation [Burrell et al., 2012]. After growth in complex medium, low concentrations of putrescine, cadaverine, spermidine, and spermine (ca. $0.05-0.1 \mu \mathrm{mol} / \mathrm{g}$ ) were detected in S. coelicolor JCM4357 [Hamana \& Matsuzaki, 1987].

In addition to de novo biosynthesis, the uptake of external polyamines can provide polyamines for bacterial cells. Since polyamines are positively charged and hydrophilic molecules at physiological $\mathrm{pH}$, they cannot pass cellular membranes by diffusion. Hence, an active transport system is required for polyamine uptake. Moreover, polyamine uptake conserves energy, since the biosynthesis of S-adenosylmethionine (AdoMet) requires ATP [Kusano \& Suzuki, 2015]. Transport of polyamines has been investigated predominantly in pathogenic bacteria, such as Proteus mirabilis, Vibrio cholerae, Streptococcus pneumonia, and Aggregatibacter actinomycetemcomitans, as well as in E. coli, which possesses several polyamine transporters [Kusano \& Suzuki, 2015]. However, the polyamine uptake in S. coelicolor is largely uninvestigated. Some candidate genes that may encode proteins for polyamine uptake were defined in an RNAseq analysis (see section Further Identification of Polyamine-Associated Genes in S. coelicolor).

\section{Polyamine Assimilation in Bacteria}

Polyamine assimilation is required to control the intracellular polyamine pool, to detoxify polyamines under excess and to utilize them as $C / N$ source under deficiency conditions. This process has been studied extensively in the Gram-negative bacteria E. coli and P. aeruginosa POA1.
It was reported that these bacteria can efficiently utilize polyamines as carbon and nitrogen sources [Kurihara et al., 2005; Yao et al., 2011; Kusano \& Suzuki, 2015]. In P. aeruginosa and E. coli, putrescine can be catabolized to succinate via the aminotransferase pathway [Schneider \& Reitzer, 2012] or the $\gamma$-glutamylation pathway [Kurihara et al., 2008; Yao et al., 2011; Schneider \& Reitzer, 2012; Krysenko et al., 2017] (shown in Fig. 6, in black). Further polyamine utilization pathways have been described in bacteria, such as the direct oxidation pathway for putrescine in P. aeruginosa (shown in Fig. 6, in orange), the spermine/ spermidine dehydrogenase pathway for spermine in $P$. aeruginosa (shown in Fig. 6, in orange), the acetylation pathway for spermidine in E. coli and B. subtilis (shown in Fig. 6, in blue) [Forouhar et al., 2005; Yao et al., 2011; Foster et al., 2013; Campilongo et al., 2014]. The different pathways and enzymes involved are shown in Figure 6.

\section{Regulation of Polyamine Assimilation Genes in Bacteria}

Since polyamines are involved in multiple bacterial functions, but can lead to cell death in excess, strict regulation of intercellular polyamine concentration at the level of biosynthesis, degradation, uptake, and efflux is required. Regulation of the polyamine-associated genes, such as those from the aminotransferase pathway and the gamma-glutamylation pathway, have been extensively studied in E. coli. It has been shown that in E. coli the gene regulation involves the alternative sigma factor $\sigma \mathrm{S}$, the alternative sigma factor for nitrogen-controlled genes $\sigma 54$, the nitrogen regulatory protein $\mathrm{C}(\mathrm{NtrC})$ and the nitrogen assimilation control protein $(\mathrm{Nac})$. The genes of the gamma-glutamylation pathway in E. coli can be regulated by the repressor PuuR, which controls the expression of $p u u$ genes in puuAP and puuDRCBE operons containing polyamine utilization genes [Partridge et al., 2006; Kusano \& Suzuki, 2015].

Regulation of the polyamine-associated genes was only recently studied also in S. coelicolor (see section Regulation of Polyamine Assimilation Genes in S. coelicolor) revealing the participation of components similar to those known from Gram-negative bacteria.

\section{Ethanolamine Metabolism in Bacteria}

\section{Distribution and Role of Ethanolamine in Bacteria}

The monoamine ethanolamine is a primary amine and primary alcohol and belongs to the class of aliphatic amino alcohols (shown in Fig. 5). Ethanolamine is a nitrog- 


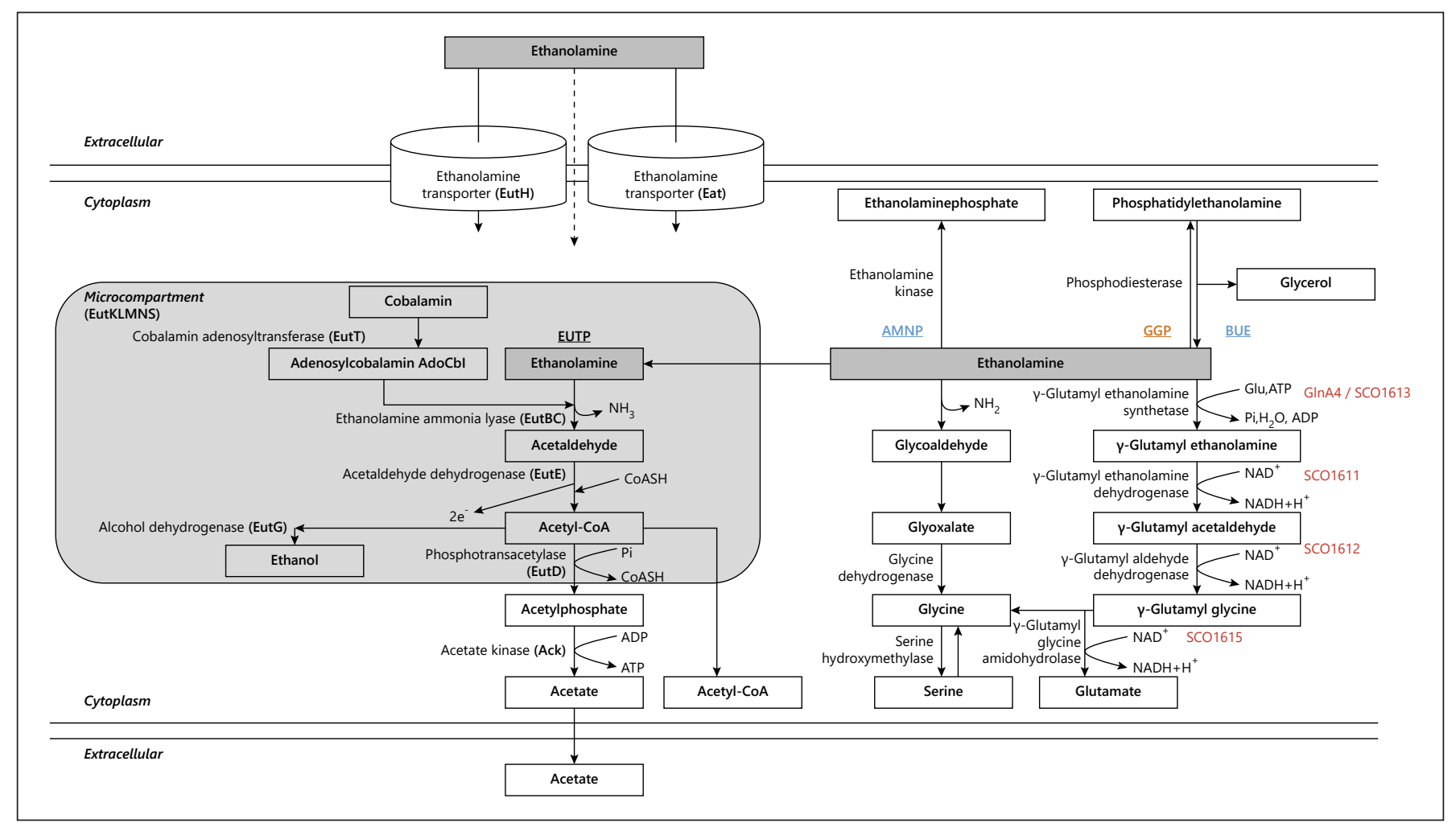

Fig. 7. Combined model of ethanolamine utilization pathways within prokaryotes [modified after Krysenko et al., 2019]. EUTP, eut-pathway; GGP, gamma-glutamylation pathway; AMP, amination pathway; BUE, biosynthetic utilization of ethanolamine. Dashed arrow represents diffusion. Pathways in black: E. coli and S. typhimurium, blue: M. tuberculosis, orange: C. salexigens, red: S. coelicolor.

enous base in phospholipids, and together with glycerol, fatty acid esters, and phosphoric acid, it is a building block of biomembranes in animals. It is a frequently occurring compound in nature as a component of cell membranes in form of phosphatidylethanolamine, which is the second most-abundant head group for phospholipids. Ethanolamine is an abundant compound in the human intestinal tract as well as in processed food [Kofoid et al., 1999; Anderson \& Kendall, 2016].

Ethanolamine has been demonstrated to be a source of carbon and nitrogen for gut-associated predominantly Gram-negative bacteria, such as Escherichia and Salmonella [Tsoy et al., 2009]. Ethanolamine utilization is a virulence determinant and contributes to a competitive advantage for intestinal pathogens [Kaval \& Garsin 2018].

Ethanolamine, its incorporation into the cell membrane and its biological role, were also studied in Grampositive bacteria such as Mycobacterium sp. [Nandedkar, 1974], Corynebacterium sp., Listeria sp., Enterococcus sp. and Clostridium sp. [Tsoy et al., 2009; Garsin, 2010], and recently in S. coelicolor [Krysenko et al., 2019].

Poly- and Monoamine Metabolism in Streptomyces coelicolor

\section{Ethanolamine Biosynthesis and Uptake in Bacteria}

The biosynthesis of ethanolamine can occur from serine. This process has been described in plants, where ethanolamine is biosynthesized from serine by decarboxylation, a process which involves a phosphatidylserine decarboxylase. As an endogenous alkalamine, ethanolamine is a principal precursor of phosphoglycerides, which are important elements in the structure of choline in biological membranes [Bingham et al., 2001]. However, in comparison to plants and mammals the biosynthesis of ethanolamine from serine has less been studied in bacteria. Similar to plants, yeast and bacteria also possess the enzyme phosphatidylserine decarboxylase for decarboxylation, but they do not synthesize ethanolamine directly as a precursor of phosphatidylserine. Instead, phosphatidylserine is predominantly synthesized in a reaction catalyzed by a phosphatidylserine synthase. The intracellularly produced phosphatidylserine can be decarboxylated into phosphatidylethanolamine, which subsequently can be cleaved by phosphodiesterases into glycerol and ethanolamine, for example during cell lysis [Cronan, 2003]. 
Extracellular ethanolamine can also pass into the cell through carrier-mediated transport or diffusion [Penrod et al., 2004]. For instance, in E. coli and S. typhimurium, ethanolamine is transported into the cell by the transport protein EutH, which is similar to permeases [Stojilikovic et al., 1995]. Most Actinobacteria and Proteobacteria can take up ethanolamine with a transporter encoded by the eat gene, which is a functional, non-homologous equivalent to eut $H$ from S. typhimurium [Tsoy et al., 2009; Gar$\sin , 2010]$.

\section{Ethanolamine Assimilation in Bacteria}

Ethanolamine utilization as a source of carbon and nitrogen has been reported in Salmonella, Escherichia, Erwinia, Flavobacterium, Klebsiella, Pseudomonas, Achromobacter, Vibrio, Escherichia as well as in Enterococcus, Arthrobacter, Mycobacterium, Corynebacterium, Clostridium [Del Papa \&Perego et al., 2008; Tsoy et al., 2009; Garsin, 2010], and in S. coelicolor [Krysenko et al., 2019].

Ethanolamine utilization in E. coli and S. typhimurium occurs in a bacterial microcompartment called the metabolosome [Kofoid et al., 1999]. In S. typhimurium and E. coli, the ethanolamine catabolic pathway includes 17 proteins encoded by genes from the ethanolamine utilization operon eut [Blackwell et al., 1976; Stojiljkovic et al., 1995; Kofoid et al., 1999]. The metabolosome is required to retain acetaldehyde, the intermediate of ethanolamine utilization. It prevents the loss of this volatile $\mathrm{C}$-source and protects the cell from the toxic effects of acetaldehyde [Penrod \& Roth, 2006]. After ethanolamine enters the cell via the ethanolamine transporter EutH or by diffusion, it reaches the metabolosome and is subsequently broken down via ethanolamine ammonia lyase (EutBC) into acetaldehyde and ammonia. The ammonia serves as a supply of reduced nitrogen, while acetaldehyde is converted into acetyl-CoA, which is used in the TCA cycle, glyoxylate cycle, and lipid biosynthesis [Garsin, 2010; Kaval \& Garsin, 2018] (shown in Fig. 7, in black).

Besides canonical ethanolamine utilization pathways that involve the metabolosome, some alternative pathways have been described. Reductive amination of glyoxate to glycine by glycine dehydrogenase has been reported in M. tuberculosis [Goldman \& Wagner, 1962]. The conversion of ethanolamine to glycine via a pathway with the intermediates glycoaldehyde and glyoxalate was reported in Mycobacterium sp. 607 [Nandedkar, 1974]. However, it was proposed that in Mycobacterium sp. ethanolamine cannot be directly utilized as a $\mathrm{C}$ - or $\mathrm{N}$-source. It can be transformed into phosphatidylethanolamine (a process also described as biosynthetic utilization of ethanolamine) [Nandedkar, 1975; Shukla \& Turner, 1980] (shown in Fig. 7, in blue).

It was also shown that diverse organisms ranging from the Actinobacteria to the Proteobacteria may possess the genetic capability for ethanolamine metabolism, which does not require eut genes. An ethanolamine glutamylation pathwaywas proposed fora $\gamma$-proteobacterium Chromohalobacter salexigens based on the EFI-GNT web tool input. In C. salexigens, ethanolamine may be glutamylated by a member of the glutamine synthase family (PF00120; ethanolamine $\gamma$-glutamylase) and subsequently reduced to L-glutamate and glycine, which are further assimilated as an N-source [Gerlt, 2016] (shown in Fig. 7, in orange).

\section{Regulation of Ethanolamine Assimilation Genes in \\ Bacteria}

Strict control of the ethanolamine utilization genes is required in order to regulate the intracellular ethanolamine concentration and to avoid its toxic effects.

Two regulatory mechanisms of ethanolamine utilization genes were studied in E. coli and S. typhimurium (the EutR system) as well as in Enterococcus faecalis (the EutVEutW system). In S. typhimurium, an EutR regulator, which belongs to the AraC family of transcriptional regulators, positively regulates the transcription of the eut operon [Roof \& Roth, 1992; Garsin, 2010]. In contrast, E. faecalis lacks the eutR gene, and the ethanolamine utilization is regulated by a two-component system composed of EutV (response regulator) and EutW (sensor histidine kinase) [Del Papa \& Perego, 2008].

It has been shown that the regulation of the eut operon may involve also other regulators. In S. typhimuri$u m$, the expression of the eut operon may be influenced by CsrA, the global regulator of invasion genes. The expression of the eut operon in E. faecalis may be affected by Fsr, the global transcriptional regulator of serine protease- and gelatinase-encoding genes [Fox et al., 2009; Garsin, 2010].

\section{Polyamine and Ethanolamine Metabolism in S. coelicolor}

Polyamine Utilization in S. coelicolor

In recent studies, a polyamine utilization pathway in S. coelicolor was postulated. It has been shown that this actinobacterium possesses a gamma-glutamylation path- 
way for polyamine utilization, which has similarities to that known in E. coli and P. aeruginosa [Krysenko et al., 2017]. The initial step of the polyamine utilization is catalyzed by the GlnA3 enzyme, which is able to glutamylate the polyamines putrescine, cadaverine, spermidine, and spermine [Krysenko et al., 2017].

Based on transcriptional studies and in silico analysis, it was possible to postulate a pathway for the subsequent steps: The glutamylated products are transformed by the predicted gamma-glutamylpolyamine oxidoreductase (SCO5671) in the second step. The SCO5671 enzyme is a close ortholog of the gamma-glutamylpolyamine oxidoreductases PuuB from E. coli and PauB1-B4 from P. aeruginosa. In the second step of the polyamine utilization pathway, SCO1281 and SCO6051 are involved. The third step of the utilization pathway is catalysed by the predicted dehydrogenases (SCO5666 and SCO5657), which are predicted homologs of the (gamma-glutamyl-) gammaaminobutyraldehyde dehydrogenases PuuC and PatD from $E$. coli. The fourth step of the pathway requires predicted hydrolases (SCO5666, SCO5657 and SCO6961) and results in the production of aminovalerate or GABA. Afterwards, the GABA aminotransferase (SCO5676) that is a predicted homolog of GabT from E. coli, catalyses the production of glutarate semialdehyde or succinate semialdehyde in the fifth step. In the last step of the polyamine utilization pathway, SCO5679, which is a predicted homolog of the succinic semialdehyde dehydrogenase GabD from E. coli, is involved, terminating the pathway by releasing succinate or glutarate and feed the tricarboxylic acid (TCA) cycle (shown in Fig. 6, see GGP). Moreover, the expression of sco5679 appeared to be induced by cadaverine, which was confirmed by RNAseq, supporting the hypothesis that SCO5679 is involved in the last step of polyamine utilization in S. coelicolor (see section Further Identification of Polyamine-Associated Genes in $S$. coelicolor).

There is a possibility of an alternative polyamine utilization pathway in S. coelicolor, since a predicted amidotransferase (SCO5655) was identified. This enzyme is an orthologue of the putrescine aminotransferase (PatA) from E. coli (shown in Fig. 6, see AMTP). Transcriptional analysis revealed that the expression of sco5655, sco6960, sco6961 was enhanced in presence of polyamines [Krysenko et al., 2017] and sco5655 was reported to be induced by a diamide [Kallifidas et al., 2010] and not by arginine [Perez-Redondo et al., 2012]. However, further analysis of SCO5655 as well as other potential aminotransferase homologs of PatA (SCO1223, SCO1284, SCO6769) are required in order to determine

Poly- and Monoamine Metabolism in Streptomyces coelicolor the functionality of the PatA-dependent pathway (shown in Fig. 6, see AMTP).

\section{Regulation of Polyamine Assimilation Genes in S. coelicolor}

The recently postulated gamma-glutamylation polyamine utilization pathway in S. coelicolor (see above) is likely to be regulated by SCO5656 (EpuRII). sco5656 is localized close to the genes that encode predicted enzymes of the polyamine utilization pathway and is annotated as putative regulator. A possible role of EpuRII in the regulation of polyamine metabolism is supported by the observation that the expression of the epuRII gene was enhanced in the presence of putrescine, cadaverine, and spermidine [Krysenko et al., 2017]. Moreover, preliminary EMSA analysis of potential regulatory targets of EpuRII revealed hints towards a complex regulation of several polyamine-associated genes, including $g \ln A 3, \operatorname{sco5676}$ (encoding a putative homolog of the 4-amino-butyrate aminotransferase GabT of $E$. coli K12), and sco5977 (encoding a putative polyamine antiporter) [Krysenko, 2018]. Further candidate genes that may encode regulators of polyamine metabolism were defined by RNAseq analysis (see section Further Identification of Polyamine-Associated Genes in S. coelicolor).

\section{Further Identification of Polyamine-Associated Genes}

in S. coelicolor

In order to identify further genes, which may encode enzymes required to metabolize polyamines or glutamylated polyamines (shown in Fig. 6), respectively, an in silico analysis was performed. Subsequently, the association of these genes to the polyamine metabolism was investigated using RT-PCR and RNAseq analysis. Expression of genes in the presence of polyamines (putrescine, cadaverine, spermidine, spermine) as the only nitrogen source was compared with their expression in the presence of ammonium. RNAseq analysis revealed significantly ( $p_{\text {adj }} \leq 0.01$, A-value: 6 ) induced expression of the amino acid transporter-encoding gene sco2724 by cadaverine, of the ammonium transporter AmtB-encoding gene $s c 05583$ by putrescine and of the transmembrane efflux protein sco3915 by spermine. Some genes (sco3435 and sco5877), whose gene products were annotated as transcriptional regulators, showed elevated expression levels in the presence of polyamines: the expression of sco3435 appeared to be induced by spermine, while the expression of $s c 05877$ by putrescine. 
Furthermore, this transcriptional analysis indicated that the expression of sco5679 is induced by cadaverine. This finding strengthened the hypothesis that sco5679 is involved in polyamine metabolism by encoding an aldehyde dehydrogenase responsible for the last step of polyamine utilization in S. coelicolor.

\section{Ethanolamine Utilization in S. coelicolor}

Studies on GS-like enzymes from S. coelicolor revealed that $\mathrm{Gln} \mathrm{A} 3$ is involved in polyamine metabolism, and GlnA2 may play a regulatory role in the nitrogen metabolism (see above). In silico analysis of GlnA4 showed low amino acid sequence similarity with known proteins involved in nitrogen metabolism but predicted structural features that differ from GlnA and other GS-like proteins. A phenotypic analysis of the $g \ln A 4$ mutant revealed growth defects in the presence of ethanolamine. Ethanolamine is glutamylated by the gamma-glutamylethanolamide synthetase GlnA4 in the first step of the utilization pathway [Krysenko et al., 2019]. Further steps of the ethanolamine utilization pathway were postulated based on in silico and transcriptional analysis. The pathway may require a predicted gamma-glutamylethanolamine dehydrogenase (SCO1611), a predicted gamma-glutamylaldehyde dehydrogenase (SCO1612) and a predicted gammaglutamylglycine amidohydrolase (SCO1615) in further reactions. Interestingly, the RNAseq analysis revealed that the scol615 gene was induced by putrescine. The pathway may end in the production of glycine and glutamate [Krysenko, 2018; Krysenko et al., 2019] (shown in Fig. 7, in red; Table 1).

\section{Regulation of Ethanolamine Assimilation Genes in \\ S. coelicolor}

In contrast to $S$. typhimurium and E. faecalis, until recently, nothing was known about the regulation of ethanolamine utilization in Actinobacteria and in S. coelicolor. In the genome of S. coelicolor, a gene sco1614 (epuRI) can be found, annotated as gene for a putative repressor and localized close to the gene $\operatorname{gln} A 4$ (sco1613). It was therefore assumed that genes encoding predicted enzymes for the ethanolamine utilization pathway may be regulated by EpuRI (SCO1614). EMSA analysis indeed revealed that $g \ln A 4$ as well as the promoter sequence of epuRI are potential targets of EpuRI [Krysenko, 2018]. The genes located downstream of $g \ln A 4$ that encode predicted enzymes of the ethanolamine utilization pathway (see above) are organized in one putative operon. Thus, these genes are most likely transcribed together with glnA4 and regulated by EpuRI. Its possible role as a nega- tive transcriptional regulator of the ethanolamine utilization associated genes was shown in a transcriptional analysis. The deletion of epuRI allowed $g \ln A 4$ expression in the presence of ammonium that does not occur in the parental strain [Krysenko et al., 2019].

\section{Conclusion}

Streptomycetes have evolved a large number of metabolic properties required for the survival under diverse environmental conditions found in soil. This is also reflected by the large genome of most streptomycetes. One of these special properties is their ability to detoxify polyamines and ethanolamine and to utilize them as $\mathrm{C} / \mathrm{N}$ sources. The key mechanism of polyamine/ethanolamine assimilation is the glutamylation of the substrate. GlnA3 and $\mathrm{G} \ln \mathrm{A} 4$, which are required for this reaction, are evolutionary derived from a universal GS enzyme. Instead of glutamylation of ammonium, GlnA3 and GlnA4 are able to glutamylate polyamines and ethanolamine in an analogous reaction. For subsequent conversions that are required for the integration of polyamines and ethanolamine into primary metabolism, candidate proteins were identified using in silico as well as transcriptional analysis. This enabled the first postulation of polyamine/ethanolamine utilization pathways in S. coelicolor. The role of these proteins in polyamine/ethanolamine metabolism will be demonstrated in biochemical analyses in the near future.

Enzymes that are able to glutamylate mono- and polyamines have been described in a small number of Gram-negative bacteria, such as E. coli (PuuA) [Kurihara et al., 2008], P. aeruginosa (PauA7) [Yao et al., 2011], and C. salexigens (PF00120 family member) [Gerlt, 2016]. These enzymes share similarities to GlnA3 and GlnA4 from S. coelicolor. Furthermore, in silico analysis showed that orthologs of GlnA3 and GlnA4 can be found in Gram-negative and Gram-positive bacteria that live and colonize specific habitats. For example, across Actinobacteria (24 genera) orthologs of GlnA3 are present in about $29 \%$ of representative species and GlnA4 in 54\% of species.

The presence of GS-like enzymes GlnA3 and GlnA4 are a special feature of streptomycetes. Only Mycobacterium sp., which has a very similar nitrogen metabolism, shares this feature. While streptomycetes have to cope with polyamines and ethanolamine in their main habitat soil, pathogenic mycobacteria are exposed to elevated polyamine concentrations during the infection process in 
macrophages. In this case, GlnA3 enables the pathogens to survive in their host. Thus, the inhibition of GlnA3 should prevent survival of Mycobacterium in macrophages and offers a novel approach to combat mycobacterial infections.

\section{Acknowledgement}

We acknowledge the support by the German Research Foundation (Deutsche Forschungsgemeinschaft, DFG) funded Research Training Group GRK1708 (I and II). Also, we would like to thank the IIT/Universität Bielefeld GmbH for the RNAseq analysis as well as the BMBF for funding part of these studies. We acknowledge the research group leader at the University of Tübingen Chambers C. Hughes for proofreading of the manuscript as well as the technical assistants Andreas Kulik, Nicole Okoniewski, and Eva Nußbaum; students Melis Girbas (Bachelor's thesis), Alena Strüder (research assistant), Maria Oswald (Master's thesis) and Moritz Bäuerle (Master's thesis) that have been involved in part of the laboratory work.

\section{Conflict of Interest Statement}

All authors declare no conflict of interest to declare.

\section{Funding Sources}

This work was supported by the DFG Research Training Group GRK1708 (I and II) and the BMBF (Fördermaßnahme "Targetvalidierung für die pharmazeutische Wirkstoffentwicklung"), project GPS-TBT (FKZ: 16 GW0183K) as well as project GSS-TUBTAR (FKZ: 16 GW0253K). S.K. was a member of the DFG Research Training Group GRK1708 (I and II).

\section{Author Contributions}

S.K. wrote the manuscript. S.K. and T.B. performed the RNAseq analysis. W.W. corrected the manuscript and provided helpful feedback of each draft of the paper. A.M. and A.B. provided helpful feedback.

\section{References}

Amin R, Franz-Wachtel M, Tiffert Y, Heberer M, Meky M, Ahmed Y, et al. Post-translational serine/threonine phosphorylation and lysine acetylation: a novel regulatory aspect of the global nitrogen response regulator $\mathrm{G} \ln \mathrm{R}$ in $\mathrm{S}$. coelicolor M145. Front Mol Biosci. 2016;3: 38.

Amin R, Reuther J, Bera A, Wohlleben W, Mast Y. A novel GlnR target gene, nnaR, is involved in nitrate/nitrite assimilation in Streptomyces coelicolor. Microbiology (Reading). 2012; 158:1172-82.

Anderson CJ, Kendall MM. Location, location, location. Salmonella senses ethanolamine to gauge distinct host environments and coordinate gene expression. Microb Cell. 2016;3: 89-91.

Behrmann I, Hillemann D, Pühler A, Strauch E, Wohlleben W. Overexpression of a Streptomyces viridochromogenes gene (glnII) encoding a glutamine synthetase similar to those of eucaryotes confers resistance against the antibiotic phosphinothricyl-alanyl-alanine. J Bacteriol. 1990;172:5326-34.

Bentley SD, Chater KF, Cerdeño-Tárraga AM, Challis GL, Thomson NR, James KD, et al. Complete Genome Sequence of the Model Actinomycete Streptomyces coelicolor A3(2). Nature. 2002;417:141-7.

Bingham E, Cohrssen B, Powell CH. Patty's Toxicology Volumes 1-9. 5th ed. New York: John Wiley \& Sons; 2001. p. 782.

Blackwell CM, Scarlett FA, Turner JM. Ethanolamine catabolism by bacteria, including Escherichia coli. Biochem Soc Trans. 1976;4:4957.

Bologna FP, Campos-Bermudez VA, Saavedra DD, Andreo CS, Drincovich MF. Character- ization of Escherichia coli EutD: a phosphotransacetylase of the ethanolamine operon. J Microbiol. 2010;48:629-36.

Burrell M, Hanfrey CC, Kinch LN, Elliott KA, Michael AJ. Evolution of a novel lysine decarboxylase in siderophore biosynthesis. Mol Microbiol. 2012;86(2):485-99.

Burrell M, Hanfrey CC, Murray EJ, Stanley-Wall NR, Michael AJ. Evolution and multiplicity of arginine decarboxylases in polyamine biosynthesis and essential role in Bacillus subtilis biofilm formation. J Biol Chem. 2010;285: 39224-38.

Campilongo R, Di Martino ML, Marcocci L, Pietrangeli P, Leuzzi A, Grossi M, et al. Molecular and functional profiling of the polyamine content in enteroinvasive $\mathrm{E}$. coli: looking into the gap between commensal $\mathrm{E}$. coli and harmful Shigella. PLoS One. 2014; 9:e106589.

Chattopadhyay MK, Tabor CW, Tabor H. Polyamines are not required for aerobic growth of Escherichia coli: preparation of a strain with deletions in all of the genes for polyamine biosynthesis. J Bacteriol. 2009;191:5549-52.

Cohen S. A Guide to the Polyamines. Oxford: Oxford University Press; 1998.

Cronan JE. Bacterial membrane lipids: Where do we stand? Annu Rev Microbiol. 2003;57:20324.

Del Papa MF, Perego M. Ethanolamine activates a sensor histidine kinase regulating its utilization in Enterococcus faecalis. J Bacteriol. 2008;190:7147-56.

Eisenberg D, Gill HS, Pfluegl GM, Rotstein SH. Structure-function relationships of glutamine synthetases. Biochim Biophys Acta. 2000; 1477:122-45.
Fink D, Falke D, Wohlleben W, Engels A. Nitrogen metabolism in Streptomyces coelicolor A3(2): modification of glutamine synthetase I by an adenylyltransferase. Microbiology (Reading). 1999;145(Pt 9):2313-22.

Fink D, Weissschuh N, Reuther J, Wohlleben W, Engels A. Two transcriptional regulators GlnR and GlnRII are involved in regulation of nitrogen metabolism in Streptomyces coelicolor A3(2). Mol Microbiol. 2002;46:331-47.

Fischer M, Alderson J, van Keulen G, White J, Sawers RG. The obligate aerobe Streptomyces coelicolor A3(2) synthesizes three active respiratory nitrate reductases. Microbiology (Reading). 2010;156:3166-79.

Forouhar F, Lee IS, Vujcic J, Vujcic S, Shen J, Vorobiev SM, et al. Structural and functional evidence for Bacillus subtilis PaiA as a novel N1spermidine/spermine acetyltransferase. J Biol Chem. 2005;280:40328-36.

Foster A, Barnes N, Speight R, Keane MA. Genomic organisation, activity and distribution analysis of the microbial putrescine oxidase degradation pathway. Syst Appl Microbiol. 2013;36:457-66.

Fox KA, Ramesh A, Stearns JE, Bourgogne A, Reyes-Jara A, Winkler WC, et al. Multiple posttranscriptional regulatory mechanisms partner to control ethanolamine utilization in Enterococcus faecalis. Proc Natl Acad Sci U S A. 2009; 106:4435-40.

Gao C, Hindra D, Mulder D, Yin C, Elliot MA. Crp is a global regulator of antibiotic production in Streptomyces. Mbio. 2012;3:e0040712.

Garsin DA. Ethanolamine Utilization in Bacterial Pathogens: Roles and Regulation. Nat Rev Microbiol. 2010;8(4):290-5. 
Gerlt JA. Tools and strategies for discovering novel enzymes and metabolic pathways. Perspect Sci. 2016;9:24-32.

Goldman DS, Wagner MJ. Enzyme systems in the mycobacteria. XIII. Glycine dehydrogenase and the glyoxylic acid cycle. Biochim Biophys Acta. 1962;65:297-306.

Hamana K, Matsuzaki S. Distribution of polyamines in actinomycetes. FEMS Microbiol Lett. 1987;41:1574-6968.

Harper C, Hayward D, Wiid I, van Helden P. Regulation of nitrogen metabolism in Mycobacterium tuberculosis: A comparison with mechanisms in Corynebacterium glutamicum and Streptomyces coelicolor. IUBMB Life. 2008;60:643-50.

Hesketh A, Fink D, Gust B, Rexer HU, Scheel B, Chater K, et al. The GlnD and GlnK homologues of Streptomyces coelicolor A3(2) are functionally dissimilar to their nitrogen regulatory system counterparts from enteric bacteria. Mol Microbiol. 2002;46:319-30.

Hillemann D, Dammann T, Hillemann A, Wohlleben W. Genetic and biochemical characterization of the two glutamine synthetases GSI and GSII of the phosphinothricyl-alanyl-alanine producer, streptomyces viridochromogenes Tü494. J Gen Microbiol. 1993;139: 1773-83.

Hodgson DA. Primary metabolism and its control in streptomycetes: a most unusual group of bacteria. Adv Microb Physiol. 2000;42:47238.

Hopwood DA. Soil to genomics: The Streptomyces chromosome. Annu Rev Genet. 2006;40: $1-23$.

Hopwood DA, Chater KF, Bibb MJ. Genetics of antibiotic production in Streptomyces coelicolor A3(2), a model streptomycete. Biotechnology. 1995;28:65-102.

Hopwood DA. Forty years of genetics with Streptomyces: From in vivo through in vitro to in silico. Microbiology (Reading). 1999;145(Pt 9):2183-202.

Kallifidas D, Thomas D, Doughty P, Paget MS The sigmaR regulon of Streptomyces coelicolor A3(2) reveals a key role in protein quality control during disulphide stress. Microbiology. 2010;156:1661-72.

Karandikar A, Sharples GP, Hobbs G. Differentiation of Streptomyces coelicolor A3(2) under nitrate limited conditions. Microbiology. 1997;143:3581-90.

Kaval KG, Garsin DA. Ethanolamine utilization in bacteria. mbio. 2018;9:e00172-12.

Kofoid E, Rappleye C, Stojiljkovic I, Roth J. The 17-gene ethanolamine (eut) operon of Salmonella typhimurium encodes five homologues of carboxysome shell proteins. J Bacteriol. 1999;181:5317-29.

Krysenko S, Matthews A, Okoniewski N, Kulik A, Girbas MG, Tsypik O, et al. Initial metabolic step of a novel ethanolamine utilization pathway and its regulation in Streptomyces coelicolor M145. mBio. 2019;10:e00326-19.

Krysenko S. Characterization of polyamine and ethanolamine utilization pathways in Strep- tomyces coelicolor M145.Tübingen: Doctora Dissertation, Eberhard Karls Universität Tübingen; 2018.

Krysenko S, Okoniewski N, Kulik A, Matthews A, Grimpo J, Wohlleben W, et al. Gamma-Glutamylpolyamine Synthetase GlnA3 is involved in the first step of polyamine degradation pathway in Streptomyces coelicolor M145. Front Microbiol. 2017;8:726.

Kumada Y, Benson DR, Hillemann D, Hosted TJ, Rochefort DA, Thompson CJ, et al. Evolution of the glutamine synthetase gene, one of the oldest existing and functioning genes. Proc Natl Acad Sci U S A. 1993;90:3009-13.

Kurihara S, Oda S, Tsuboi Y, Kim HG, Oshida M, Kumagai $\mathrm{H}$, et al. gamma-Glutamylputrescine synthetase in the putrescine utilization pathway of Escherichia coli K-12. J Biol Chem. 2008;283:19981-90.

Kurihara S, Oda S, Kato K, Kim HG, Koyanagi T, Kumagai $\mathrm{H}$, et al. A novel putrescine utilization pathway involves gamma-glutamylated intermediates of Escherichia coli K-12. J Biol Chem. 2005;280:4602-8.

Magasanik B. Genetic control of nitrogen assimilation in bacteria. Annu Rev Genet. 1982;16: 135-68.

Merrick MJ, Edwards RA. Nitrogen control in bacteria. Microbiol Rev. 1995;59:604-22.

Miller-Fleming L, Olin-Sandoval V, Campbell K, Ralser M. Remaining Mysteries of Molecular Biology: The Role of Polyamines in the Cell. J Mol Biol. 2015;427:3389-406.

Nakada Y, Itoh Y. Identification of the putrescine biosynthetic genes in Pseudomonas aeruginosa and characterization of agmatine deiminase and $\mathrm{N}$-carbamoylputrescine amidohydrolase of the arginine decarboxylase pathway. Microbiology (Reading). 2003; 149: 707-14.

Nandedkar AK. Biosynthesis of phosphatidyl ethanolamine in Mycobacterium 607. Biochem Med. 1975;12:116-22.

Nandedkar AK. Report on the utilization of ethanolamine-1-14C by Mycobacterium 607. Biochem Med. 1974;11:67-70.

Nentwich M. Die Funktion von GlnA2 in der transkriptionellen und posttranslationalen Kontrolle des Stickstoffmetabolismus in Streptomyces coelicolor M145. Doctoral Dissertation. Tübingen: Eberhard Karls Universität Tübingen; 2012.

Ninfa AJ, Jiang P, Atkinson MR, Peliska JA. Integration of antagonistic signals in the regulation of nitrogen assimilation in Escherichia coli. Curr Top Cell Regul. 2001;36:31-75.

Oshima T, Hamasaki N, Uzawa T. Biochemical Properties of Unusual Polyamines Found in an Extreme Thermophile, Thermus thermophiles. In: Zappia V, Pegg AE, editors. Progress in Polyamine Research. Boston: Springer; 1988. p. 633-42

Partridge JD, Scott C, Tang Y, Poole RK, Green J. Escherichia coli transcriptome dynamics during the transition from anaerobic to aerobic conditions. J Biol Chem. 2006;281:2780615.
Penrod JT, Mace CC, Roth JR. A pH-sensitive function and phenotype: evidence that $\mathrm{EutH}$ facilitates diffusion of uncharged ethanolamine in Salmonella enterica. J Bacteriol. 2004;186:6885-90.

Penrod JT, Roth JR. Conserving a volatile metabolite: a role for carboxysome-like organelles in Salmonella enterica. J Bacteriol. 2006;188: 2865-74.

Perez-Redondo R, Rodriguez-Garcia A, Botas A, Santamarta I, Martin JF, Liras P. ArgR of Streptomyces coelicolor is a versatile regulator. PLoS One. 2012;7:e32697.

Pesole G, Gissi C, Lanave C, Saccone C. Glutamine synthetase gene evolution in bacteria. Mol Biol Evol. 1995;12:189-97.

Reuther J, Wohlleben W. Nitrogen Metabolism in Streptomyces coelicolor: Transcriptional and Post-Translational Regulation. J Mol Microbiol Biotechnol. 2007;12:139-46.

Rexer HU, Schäberle T, Wohlleben W, Engels A Investigation of the functional properties and regulation of three glutamine synthetase like genes in Streptomyces coelicolor A3(2). Arch Microbiol. 2006;186:447-58.

Rodríguez-García A, Sola-Landa A, Apel K, Santos-Beneit F, Martín JF. Phosphate control over nitrogen metabolism in Streptomyces coelicolor: Direct and indirect negative control of glnR, gln A, glnII and amtB expression by the response regulator PhoP. Nucleic Acids Res. 2009;37:3230-42.

Roof DM, Roth JR. Autogenous regulation of ethanolamine utilization by a transcriptional activator of the eut operon in Salmonella typhimurium. J Bacteriol. 1992;174:663443.

Santos-Beneit F, Rodríguez-García A, Martín JF. Overlapping binding of PhoP and AfsR to the promoter region of glnR in Streptomyces coelicolor. Microbiol Res. 2012;167: $532-5$.

Schneider BL, Reitzer L. Pathway and enzyme redundancy in putrescine catabolism in Escherichia coli. J Bacteriol. 2012;194:4080-8.

Shukla SD, Turner JM. Microbial metabolism of amino alcohols. Biosynthetic utilization of ethanolamine for lipid synthesis by bacteria. Biochem J. 1980;186:13-9.

Sola-Landa A, Rodríguez-García A, Amin R, Wohlleben W, Martín JF. Competition between the GlnR and PhoP regulators for the gln $\mathrm{A}$ and $\mathrm{amtB}$ promoters in Streptomyces coelicolor. Nucleic Acids Res. 2013;41:176782.

Stojiljkovic I, Baumler AJ, Heffron F. Ethanolamine utilization in Salmonella typhimurium: nucleotide sequence, protein expression, and mutational analysis of the $\operatorname{cch} A, \operatorname{cchB}$, eutE, eutJ, eutG, eutH gene cluster. J Bacteriol. 1995;177:1357-66.

Suzuki H, Kurihara S. Polyamine Catabolism in Prokaryotes. In: Kusano T, Suzuki H, editors. Polyamines: A Universal Molecular Nexus for Growth, Survival, and Specialized Metabolism. Tokyo: Springer; 2015. p. 4759. 
Thomas GH, Mullins JG, Merrick M. Membrane topology of the Mep/Amt family of ammonium transporters. Mol Microbiol. 2000;37: 331-44.

Tiffert Y, Supra P, Wurm R, Wohlleben W, Wagner R, Reuther J. The Streptomyces coelicolor GlnR regulon: identification of new GlnR targets and evidence for a central role of GlnR in nitrogen metabolism in actinomycetes. Mol Microbiol. 2008;67:861-80.

Tomar PC, Lakra N, Mishra SN. Cadaverine: a lysine catabolite involved in plant growth and development. Plant Signal Behav. 2013;8: 25850.

Tsoy O, Ravcheev D, Mushegian A. Comparative genomics of ethanolamine utilization. J Bacteriol. 2009;191:7157-64.
Wang J, Zhao GP. GlnR positively regulates nasA transcription in Streptomyces coelicolor. Biochem Biophys Res Commun. 2009;386:7781.

Wang R, Mast Y, Wang J, Zhang W, Zhao G, Wohlleben $\mathrm{W}$, et al. Identification of twocomponent system AfsQ1/Q2 regulon and its cross-regulation with GlnR in Streptomyces coelicolor. Mol Microbiol. 2013;87:30-48.

Wray LV, Jr, Fisher SH. The Streptomyces coelicolor glnR gene encodes a protein similar to other bacterial response regulators. Gene. 1993;130:145-50.
Wray LV, Fisher SH. Cloning and nucleotide sequence of the Streptomyces coelicolor gene encoding glutamine synthetase. Gene. 1988; 71:247-56.

Yao X, He W, Lu CD. Functional characterization of seven $\gamma$-Glutamylpolyamine synthetase genes and the bauRABCD locus for polyamine and $\beta$-Alanine utilization in Pseudomonas aeruginosa PAO1. J Bacteriol. 2011; 193:3923-30.

Yeates TO, Kerfeld CA, Heinhorst S, Cannon GC, Shively JM. Protein based organelles in bacteria: carboxysomes and related microcompartments. Nat Rev Microbiol. 2008;6:681-91.

Young CC, Chen LF. Polyamines in humic acid and their effect on radical growth of lettuce seedlings. Plant Soil. 1997;195(1):143-9. 